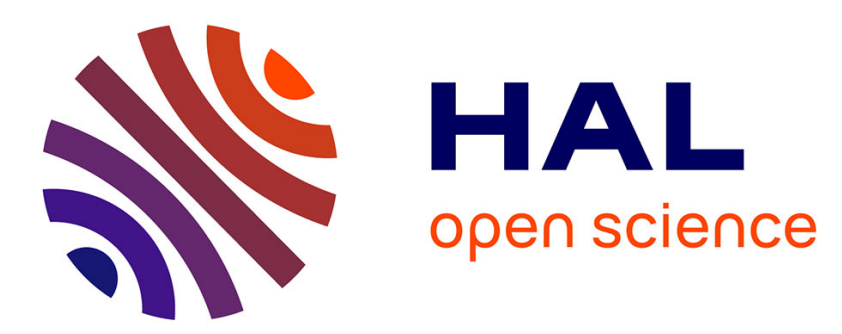

\title{
Arbitrary vortex arrays realized from optical winding of frustrated chiral liquid crystals
}

\author{
Bin Yang, Etienne Brasselet
}

\section{To cite this version:}

Bin Yang, Etienne Brasselet. Arbitrary vortex arrays realized from optical winding of frustrated chiral liquid crystals. Journal of Optics, 2013, 15 (4), pp.044021. 10.1088/2040-8978/15/4/044021 . hal-00814243

\section{HAL Id: hal-00814243 \\ https://hal.science/hal-00814243}

Submitted on 9 Mar 2018

HAL is a multi-disciplinary open access archive for the deposit and dissemination of scientific research documents, whether they are published or not. The documents may come from teaching and research institutions in France or abroad, or from public or private research centers.
L'archive ouverte pluridisciplinaire HAL, est destinée au dépôt et à la diffusion de documents scientifiques de niveau recherche, publiés ou non, émanant des établissements d'enseignement et de recherche français ou étrangers, des laboratoires publics ou privés.

\section{(1)(1) $\$(0)$}

Distributed under a Creative Commons Attribution - NonCommercial - ShareAlikel 4.0 


\title{
Arbitrary vortex arrays realized from optical winding of frustrated chiral liquid crystals
}

\author{
Bin Yang and Etienne Brasselet \\ Univ. Bordeaux, Laboratoire Ondes et Matière d'Aquitaine, UMR 5798, F-33400 Talence, France \\ and \\ CNRS, Laboratoire Ondes et Matière d'Aquitaine, UMR 5798, F-33400 Talence, France \\ E-mail: e.brasselet@1oma.u-bordeaux1.fr
}

\begin{abstract}
We report on the realization of on-demand reconfigurable arbitrary arrays of microscopic optical vortex generators in chiral liquid crystals. These generators are optically inscribed in frustrated cholesteric films by means of the laser-induced local winding of the chiral liquid crystal mesophase. This leads to the storage of microscopic, metastable, topological defect structures endowed with space-variant birefringent properties. Such structures are shown to produce optical vortices with a well-defined orbital state as a result of a spin-to-orbital angular momentum conversion process.
\end{abstract}

Keywords: optics of liquid crystals, topological liquid crystal defects, optical vortex generation

The orbital angular momentum of light is associated with the phase spatial distribution of an optical field. A particular case corresponds to light beams endowed with phase singularities, commonly called optical vortices. Among existing techniques for producing them, imprinting the topological information of a natural material topological defect into a light field has the advantage of relying on the self-engineering of matter. This allows one to consider nanoscale spatial resolution without the need for machining techniques. Liquid crystals are well suited for this purpose as already demonstrated by using dislocations [1], hedgehog point defects [2] and umbilical defects $[3,4]$.

Still, obtaining reconfigurable dense and large assemblies of optical vortex generators from liquid crystals remains a challenging issue. Strategies based on laser-induced defect structures are attractive as regards this aim. In a previous work, on-demand optical vortex generation in achiral nematic liquid crystal films using paraxial Gaussian beams has been demonstrated experimentally [5]. However, the light-induced nonsingular topological structures at work relax once the writing beam is turned off, thereby preventing the simple realization of arbitrary arrays of optical vortex generators. Instead, here we propose to use a chiral nematic phase-a cholesteric liquid crystal.

The cholesteric phase is a nematic phase whose director $\mathbf{n}$, a unit vector that defines the local averaged molecular orientation, twists in a well-defined direction. It is generally prepared by dissolving a chiral dopant in a nematic host, the resulting mixture being characterized by its helical pitch $p$, i.e. the distance over which $\mathbf{n}$ rotates by $2 \pi$; see figure 1 . When the intrinsic helical order is incompatible with the orientational boundary conditions, frustration arises [6]. A typical example is the case of a cholesteric film with orientational boundary conditions that are perpendicular to the plane of the film. For thin enough samples compared to the pitch, the director field at rest can be unwound [7], as illustrated in figure 1 where $\mathbf{n}_{0}$ defines the uniform orientational ground state.

Field-induced defect structures can be obtained in such frustrated cholesteric films, for instance by applying a uniform 


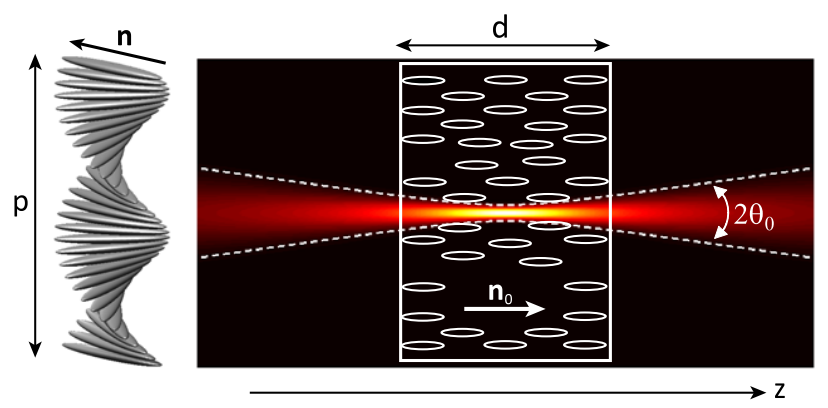

Figure 1. Illustration of the experiment. A frustrated cholesteric liquid crystal film with pitch $p$, thickness $d$ and perpendicular orientational boundary conditions is illuminated at normal incidence with a paraxial Gaussian laser beam with divergence $\theta_{0}$ (in the air). Since $d / p \lesssim 1$, the orientational ground state for the director field, $\mathbf{n}_{0}$, is unwound.

electric field. An early example is the case of the so-called 'spherulites'; see [8] for a review. Localized structures dubbed 'torons' can also be generated by electromagnetic fields, as shown in [9] where tightly focused Laguerre-Gaussian laser beams have been used.

Here we report on reconfigurable arbitrary arrays of microscopic optical vortex generators made of defect structures in frustrated cholesteric films. Such structures result from the local optical winding of the cholesteric induced by paraxial Gaussian beams. As observed in the nonparaxial case [9], the winding process consists of a sequence of two kinds or reordering transitions. Here we quantitatively identify the first one as the so-called optical Fréedericksz transition (OFT) [10] whereas the second one leads to topological anisotropic structures allowing for the production of large and dense assemblies of polarization controlled optical vortices owing to the spin-orbit interaction of light.

The sample is prepared from the nematic 5CB doped by the chiral dopant S811 (both from Merck) at $0.36 \%$ weight, which gives $p=18 \mu \mathrm{m}$ [11]. This mixture is sandwiched between two glass substrates provided with perpendicular anchoring conditions and spaced by $d=15 \mu \mathrm{m}$. The director field is therefore totally unwound at rest. A linearly polarized Gaussian beam at $532 \mathrm{~nm}$ wavelength and with divergence $\theta_{0}=12.7^{\circ} \pm 0.3^{\circ}$ in the air impinges at normal incidence onto the film; see figure 1 .

The optically wound structures are characterized using very low light intensity uniform illumination, hence discarding any spurious contribution from the probe field to the observed director distortion. As expected, a uniform dark state is observed between crossed linear polarizers at zero pump beam power, when $\mathbf{n}=\mathbf{n}_{0}$. In contrast, a bright state is observed above a first threshold power $P=P_{\text {th }, 1} \simeq 20 \mathrm{~mW}$ from which we conclude to the optical reorientation of the director, $\mathbf{n} \neq \mathbf{n}_{0}$. Such a light-induced distorted state relaxes to the ground state when the writing beam is turned off as $P<P_{\text {th }, 2} \simeq 35 \mathrm{~mW}$. In contrast, a residual distorted state is found above this second threshold. This is summarized in figure 2 which displays the diameter $D$ of the light-induced structure as a function of $P$. In practice, $D$ is defined as twice the radius that corresponds to $10 \%$ of the last maximum of



Figure 2. Diameter $D$ of the light-induced reoriented director field versus the incident total power $P$. Above the threshold power $P=P_{\text {th }, 2}$, the top markers refer to the steady state under illumination whereas the bottom ones correspond to the residual state after the writing beam is turned off.

the probe intensity profiles. A remarkable feature of figure 2 is the abrupt increase of $D$ at $P=P_{\text {th }, 2}$, which is associated with a structural transition. Indeed, distinct director fields are observed just below and above the threshold, as illustrated by figures 3(a) and (b), respectively. In addition, the residual state obtained after turning off the light beam initially set at $P \gtrsim P_{\mathrm{th}, 2}$ is shown in figure 3(c). Its structure differs from the steady-state one and its diameter is always found to be smaller than the steady-state value whatever the value of $P>P_{\text {th }, 2}$, as shown in figure 2.

Noticeably, the intensity pattern between crossed linear polarizers below the threshold power $P_{\text {th,2 }}$ exhibits a dark cross pattern. This is the indication of an axisymmetric reorientation profile. It could be argued that such an observation results from thermal effects. Indeed local melting of the liquid crystal would lead to an isotropic droplet that produces exactly this type of intensity pattern. However, by solving the heating problem (for instance following the analytical formulation given in [12]) and taking a typical absorption value of $0.05 \mathrm{~cm}^{-1}$ for the liquid crystal mixture, we find a maximal temperature elevation of the order of $1 \mathrm{~K}$. Noting that the experiments were performed at room temperature $\left(T \sim 20-25^{\circ} \mathrm{C}\right)$, this cannot be explained either by the existence of a nematic-to-isotropic transition (the $5 \mathrm{CB}$ melting point is $\sim 35^{\circ} \mathrm{C}$ ) or by the ensuing modifications of the elastic constants (a variation of a few per cent is expected [13, 14]). Another explanation could rely on the athermal lowering of the liquid crystal order parameter as a result of light-induced reorientation gradients, which would play a similar role to the presence of an isotropic droplet. An alternative qualitative explanation can also be proposed, recalling that the experiments are made using $\theta_{0} \sim 100 \mathrm{mrad}$. 

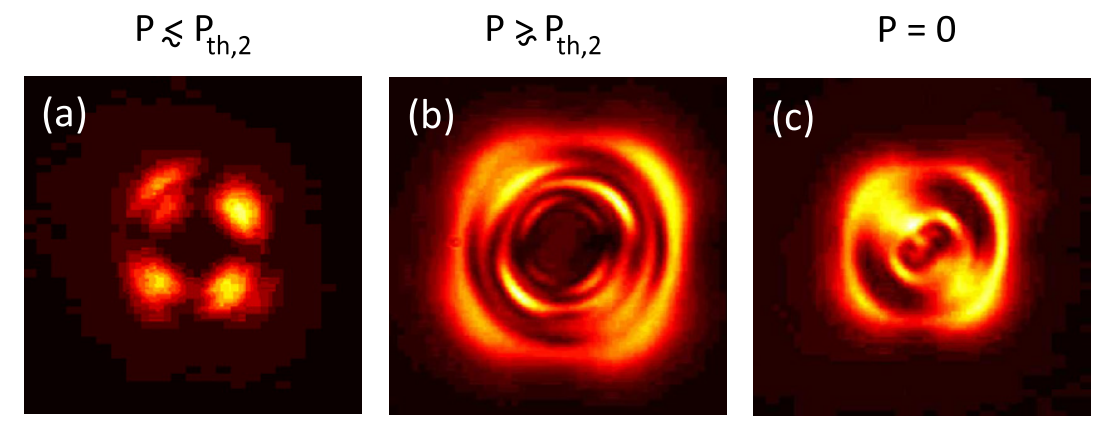

$20 \mu \mathrm{m}$

Figure 3. Illustration of the abrupt change of the diameter and orientational state of the laser-induced reoriented structure near to $P=P_{\text {th }, 2}$; see figure 2. ((a), (b)) False color representation of the steady-state structure just below/above the threshold observed using a $632.8 \mathrm{~nm}$ probe wavelength. (c) Residual state after the writing beam is turned off. Observations are carried out between crossed linear polarizers.

This suggests a topological optical reorientation to be at work - a process leading to localized nonsingular topological defect structures originally introduced for nematic films illuminated by Gaussian beams at normal incidence $[15,16]$. This would lead to complex director reorientation rather than in-plane distortions, as usually described in the standard OFT picture [17]. Still, hereafter we show that $P_{\text {th,1 }}$ quantitatively corresponds to the OFT threshold power.

For this purpose let us introduce the OFT intensity threshold for a plane wave illumination (referred to as ' $\infty$ ') at normal incidence of a nematic film of thickness $L, I_{\text {nem }}^{\infty}=$ $\pi^{2} c K_{3} n_{\|}^{2} /\left[L^{2} n_{\perp}\left(n_{\|}^{2}-n_{\perp}^{2}\right)\right]$ [18], where $c$ is the speed of light and $K_{3}$ is the bend elastic constant. For frustrated cholesterics, that is $d / p<1 /\left(2 k_{2}\right)$ where $k_{2}=K_{2} / K_{3}$ with $K_{2}$ the twist elastic constant, the OFT threshold intensity is reduced by a factor $f_{\text {chol }}=1-\left(2 k_{2} d / p\right)^{2}$ with respect to that for nematics [19]. In a real experiment, however, finite beam size effects are expected. For Gaussian beams, the on-axis OFT threshold intensity is increased by a factor $f_{\text {beam }}=\left[1+L \sqrt{2} /\left(\pi w_{0}\right)\right]^{2}[20]$, where $w_{0}$ is the beam waist radius at $\exp (-2)$ of its maximum intensity in the focal plane that is located in the present case in the middle of the film; see figure 1. The resulting expression for the OFT threshold power for Gaussian beams normally incident on a frustrated cholesteric film is therefore $P_{\mathrm{OFT}}=\left(\pi w_{0}^{2} / 2\right) f_{\text {chol }} f_{\text {beam }} I_{\text {nem }}^{\infty}$, namely

$P_{\mathrm{OFT}}=\frac{\pi^{3} c K_{3} n_{\|}^{2}}{2 n_{\perp}\left(n_{\|}^{2}-n_{\perp}^{2}\right)}\left[1-\left(\frac{2 k_{2} d}{p}\right)^{2}\right]\left(\frac{w_{0}}{L}+\frac{\sqrt{2}}{\pi}\right)^{2}$.

Using the typical value $k_{2}=0.4$, one finds from equation (1) $P_{\mathrm{OFT}}=P_{\mathrm{th}, 1}$ for $K_{3} \simeq 9 \mathrm{pN}$, which corresponds to the data measured for $5 \mathrm{CB}$ at $21^{\circ} \mathrm{C}$ in [13] and at $20^{\circ} \mathrm{C}$ in [14]. $P_{\text {th, } 1}$ thus quantitatively corresponds to the OFT under the finite-size excitation beam, thereby validating quantitatively previous qualitative statements [21] and supporting the notion of a winding transition driven by optical reorientation rather than thermal or anchoring effects.

The diameter at the onset of the second transition, like that of the residual structure, satisfies $D^{*} / p \simeq 1.2$, at least for not too high input power; see figure 2 . This is a value close to the critical ratio $(d / p)^{*}=1 /\left(2 k_{2}\right)$ above which the ground state $\mathbf{n}_{0}$ is unstable in the absence of external fields [22]. Indeed, $k_{2}=0.4$ gives $(d / p)^{*}=1.25$. Such a value agrees with the topological description of spherulites proposed by Pirkl et al [23]. Moreover, we point out that the three-dimensional structure of the latter spherulites is the same as the one experimentally determined for the T3-1 toron in [9], as one can judge by comparing figure 11 of [23] with figure 3 of [9] - an equivalence seemingly missed so far.

Light-induced spherulites enable one to twist the wavefront of the light itself, thereby producing optical orbital angular momentum. Indeed a phase singularity with topological charge 2 (i.e. the phase winds by $2 \times 2 \pi$ along a closed circuit around the singularity) can be generated from spherulites when using a circularly polarized read-out field owing to the space-variant birefringent nature [24]. The underlying spin-to-orbital angular momentum conversion process can be grasped by considering the propagation of a normally incident circularly polarized plane wave through an inhomogeneous and anisotropic axisymmetric waveplate whose optical axis makes an angle $\phi+\varphi(r)$, where $(r, \phi)$ are polar coordinates in the $(x, y)$ plane and $\Delta(r)$ refers to the local axisymmetric birefringent phase retardance profile. Let us consider the incident electric field $\mathbf{E}_{\text {in }}=E_{0} \mathrm{e}^{-\mathrm{i}\left(\omega t-k_{0} z\right)} \mathbf{e}_{\sigma}$ where $\omega$ is the light angular frequency at time $t, k_{0}$ is the wavevector in vacuum and $\mathbf{e}_{\sigma}=\left(\mathbf{e}_{x}+\mathrm{i} \sigma \mathbf{e}_{y}\right) / \sqrt{2}$ is the unit vector that defines the polarization state, with $\sigma= \pm 1$ for left/right handedness. Neglecting diffraction effects, the output light field can be calculated using the Jones formalism:

$$
\begin{aligned}
\mathbf{E}_{\text {out }}(r, \phi) \propto & E_{0}\left\{\cos [\Delta(r) / 2] \mathbf{e}_{\sigma}\right. \\
& \left.+\mathrm{i} \sin [\Delta(r) / 2] \mathrm{e}^{\mathrm{i} 2 \sigma[\phi+\varphi(r)]} \mathbf{e}_{-\sigma}\right\} .
\end{aligned}
$$

From the phase term $\exp (\mathrm{i} 2 \sigma \phi)$ in the above equation, one recognizes a phase singularity with topological charge $\ell=$ $2 \sigma$ embedded in the contra-circularly polarized output field component with respect to the incident polarization state. As a consequence, the intensity profile of the vortex field component associated with the polarization state $\mathbf{e}_{-\sigma}, I \propto$ $\sin ^{2}[\Delta(r) / 2]$, has null on-axis intensity (see later: figure $5(\mathrm{~d})$ ). That is to say, the light-induced spherulite is characterized by the on-axis director pointing along the $z$ axis, $\Delta(r)=0$, which follows from axisymmetry. 
(a)

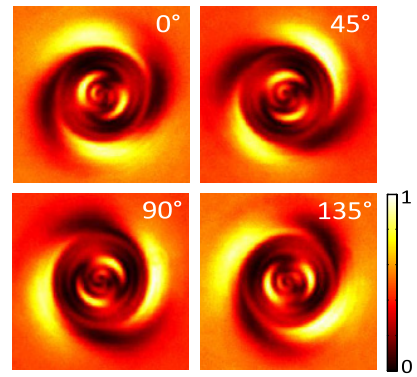

(b)

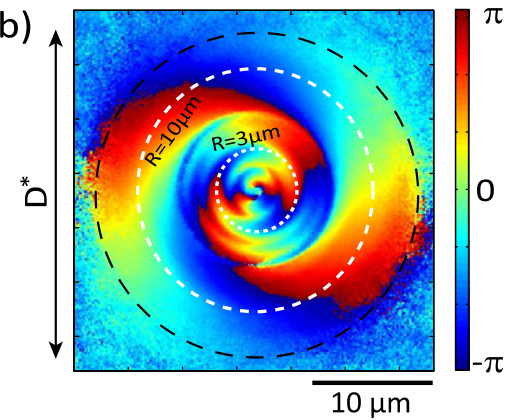

(c)

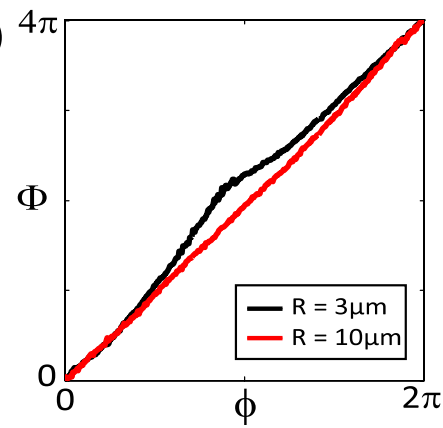

Figure 4. Optical vortex generation. (a) Normalized intensity spatial distribution $I_{\alpha}$ with $\alpha=0^{\circ}, 45^{\circ}, 90^{\circ}$ and $135^{\circ}$ under circularly polarized monochromatic illumination; see the text for details. (b) Phase spatial distribution of the contra-circularly polarized component of the output field with respect to the circular polarization state of the incident probe beam deduced from the data shown in panel (a).

(c) Azimuthal phase profile along circles of different radii that reveal a phase singularity with topological charge 2.

The generation of an optical phase singularity is a passive process that does not depend on the wavelength, though its efficiency depends on the birefringent phase retardance which obviously depends on wavelength. Experimentally, it is possible to observe the phase singularity produced by noting that the spatial distribution of the orientation of the polarization ellipse major axis of the total output field, $\psi(x, y)$, depends linearly on the phase difference between the right-handed and left-handed circular field components,

$$
\psi(x, y)=\left[\arg \left(\mathbf{E}_{\text {out }} \cdot \mathbf{e}_{+1}\right)-\arg \left(\mathbf{E}_{\text {out }} \cdot \mathbf{e}_{-1}\right)\right] / 2 .
$$

The azimuthal phase distribution $\Phi(x, y)$ of the $\mathbf{e}_{-\sigma}$-polarized component of the output field is therefore given by the simple relationship

$$
\Phi=2 \psi .
$$

From the standard polarimetry relationship $\psi=(1 / 2)$ $\arctan \left[\left(I_{+45^{\circ}}-I_{-45^{\circ}}\right) /\left(I_{0^{\circ}}-I_{90^{\circ}}\right)\right][25]$, where $I_{\alpha}$ refers to the intensity distribution obtained by placing a polarizer at an angle $\alpha$ from the $x$ axis at the output of the film (see figure 4(a)), the phase map of the optical vortex can be measured. The result is displayed in figure 4(b). A $4 \pi$ phase change per full rotation around the center of the spherulite is observed whichever closed circuit around the center of the spherulite is chosen. We thus conclude the generation of an optical vortex with topological charge 2 . This is illustrated in figure 4(c) where we show the dependence of $\Phi$ on $\phi$ along circles of radius $R=3 \mu \mathrm{m}$ (white short dashed in figure 4(b)) and $10 \mu \mathrm{m}$ (white dashed in figure 4(b)). In addition, the area outside which the phase cannot be determined as a result of negligible director reorientation has a diameter that corresponds to the residual state diameter $D^{*}$; see figure 4(b).

A finer description of the phase map should take into account diffraction effects associated with the presence of topological liquid crystal defects, namely a nonsingular disclination ring with typical diameter $\sim p / 2$ and on-axis point defects $[23,9]$. This is however beyond the scope of the present work. Nevertheless, it is worth mentioning that the existence of a radially varying phase profile of the vortex field component described by the term $\exp [\mathrm{i} 2 \sigma \varphi(r)]$ in equation (2) is retrieved by our observations, as seen in figure 4(b) as a swirl of the azimuthal phase profile.

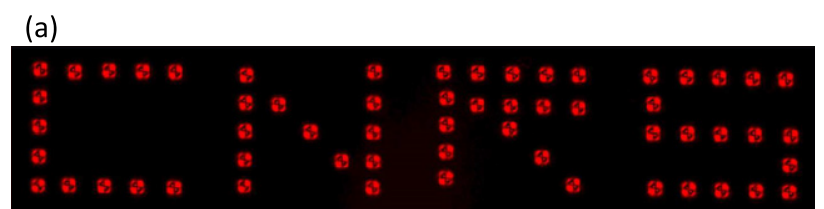

(b)

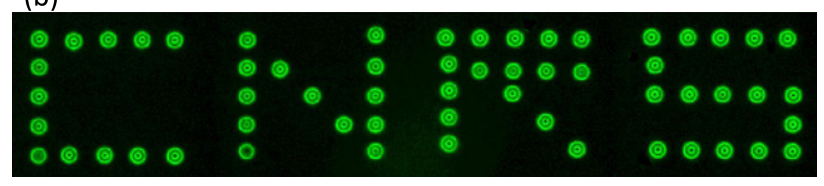

(c)

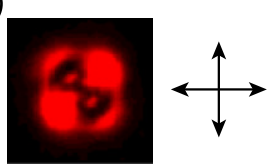

(d)

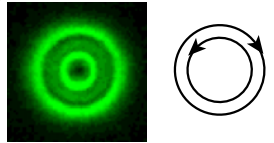

$100 \mu \mathrm{m}$

Figure 5. Demonstration of on-demand direct laser writing of arbitrary arrays of optical vortices. The four-letter acronym CNRS is observed between crossed linear polarizers at $633 \mathrm{~nm}$ wavelength (a) and between crossed circular polarizers at $532 \mathrm{~nm}$ wavelength (b). The enlargement of an individual pixel of each type is shown in panels (c) and (d), respectively, where arrows refer to the orientation of the polarizers.

Since laser-induced spherulites are metastable states, arbitrary arrays of optical vortex generators can be stored in frustrated cholesteric films. This is illustrated in figure 5 where the four-letter logo 'CNRS' is displayed, each pixel being a charge-two optical vortex when observed between crossed circular polarizers. In this figure, two reading schemes are presented. In figure 5(a) the pattern is observed between crossed linear polarizers at $633 \mathrm{~nm}$ wavelength, whereas it is observed between crossed circular polarizers at $532 \mathrm{~nm}$ wavelength in figure $5(\mathrm{~b})$, the corresponding pixels being shown in figures $5(\mathrm{c})$ and $(\mathrm{d})$.

Moreover, any assembly of optical vortex generators can be erased, either locally or globally. From the local point of view, slightly off-axis illumination of a structure by the writing beam itself leads to its erasure. It involves laser-induced orientational destabilization and has a threshold character, a beam with power $P \ll P_{\mathrm{th}, 2}$ having no effect. Global erasure can be obtained as well by applying a low-voltage quasistatic electric field along the normal to 
the film, typically a few volts, a value that depends on the dielectric anisotropy of the liquid crystal mixture. This is made possible owing to a positive dielectric anisotropy, which leads to an electrical torque density exerted on the medium that tends to align the director field along the electric field (here the $z$ axis). The director field is eventually unwound, thereby restoring the uniform ground state $\mathbf{n}_{0}$ above a threshold voltage, as previously shown in the case of localized structures generated by tightly focused infrared laser beam [26]. If a quantitative estimation of the characteristic time of the erasure process is a difficult task that should account for the presence of defects, it is qualitatively expected to scale as the orientational relaxation time of (achiral) nematic films, namely $\tau=\gamma_{1} L^{2} /\left(\pi^{2} K\right)$ where $\gamma_{1}$ is the orientational viscosity and $K$ the typical value of the elastic constants, which is of the order of $10 \mathrm{~ms}$ for the present film thickness. Obviously a field dependent correcting factor should be introduced since the higher the applied voltage, the smaller the erasing time.

Finally we stress that the present switchable microscopic optical vortex generators created by light in frustrated cholesterics with pitch $p$ have typical cross-section $p^{2}$. This is much less than what was recently reported in [27], where the strategy is to create diffraction gratings endowed with fork singularities in order to generate optical vortices. Indeed the finger defect structures used in the latter reference have typical cross-section $N p^{2}$ with $N>1000$. Such a drastic downsizing of the required spatial dimensions is made possible by exploiting the optical anisotropy rather than the refractive properties of the light-induced defect structures.

To conclude, paraxial Gaussian beams enable the local optical winding of frustrated cholesteric liquid crystal films. The resulting localized space-variant birefringent structures can be used to create arbitrary arrays of microscopic optical vortex generators endowed with write-and-erase capabilities. This extends the use of liquid crystal defect structures to on-demand integrated singular photonic applications that have been restricted so far to the realization of multistable photonic architectures [9] and electrically tunable microlens arrays [28]. In view of the great application potential of spinto-orbital angular momentum conversion in photonics [29], the present results might be useful towards the elaboration of large-scale singular microphotonics elements.

\section{References}

[1] Voloschenko D and Lavrentovich O D 2000 Opt. Lett. 25317

[2] Brasselet E, Murazawa N, Misawa H and Juodkazis S 2009 Phys. Rev. Lett. 103103903

[3] Brasselet E and Loussert C 2011 Opt. Lett. 36719

[4] Brasselet E 2012 Phys. Rev. Lett. 108087801

[5] Brasselet E 2010 Phys. Rev. A 82063836

[6] Kamien R D and Selinger J V 2001 J. Phys.: Condens. Matter $13 \mathrm{R} 1$

[7] de Gennes P G and Prost J 1995 The Physics of Liquid Crystals 2nd edn (Oxford: Clarendon)

[8] Oswald P, Baudry J and Pirkl S 2000 Phys. Rep. 33767

[9] Smalyukh I I, Lansac Y, Clark N A and Trivedi R P 2010 Nature Mater. 9139

[10] Tabiryan N V, Sukhov A V and Zel'dovich B Y 1986 Mol. Cryst. Liq. Cryst. 1361

[11] Gvozdovskyy Y and Lisetski L N 2007 Funct. Mater. 14332

[12] Miroshnichenko A E, Brasselet E and Kivshar Y S 2008 Phys. Rev. A 78053823

[13] Bunning J D, Faber T E and Sherell P L 1981 J. Phys. 421175

[14] Madhusudana N V and Pratibha R 1982 Mol. Cryst. Liq. Cryst. 89249

[15] Brasselet E 2009 Opt. Lett. 343229

[16] Brasselet E 2010 J. Opt. 12124005

[17] Zolot'ko A S, Kitaeva V F, Kroo N, Sobolev N N and Chillag L 1980 JETP Lett. 32158

[18] Zel'dovich B Ya, Tabiryan N V and Chilingaryan Yu S 1981 Sov. Phys.-JETP 5432

[19] Maddalena P, Arnone G, Abbate G, Marrucci L and Santamato E 1995 Mol. Cryst. Liq. Cryst. 261113

[20] Zolot'ko A S, Kitaeva V F, Kuyumchyan V A, Sobolev N and Sukhorukov A P 1982 JETP Lett. 3680

[21] Smalyukh I I, Kaputa D, Kachynski A V, Kuzmin A N, Ackerman P J, Twombly C W, Lee T, Trivedi R P and Prasad P N 2012 Opt. Express 206870

[22] Zel'dovich B Ya and Tabiryan N V 1981 JETP Lett. 34406

[23] Pirkl S, Ribière P and Oswald P 1993 Liq. Cryst. 13413

[24] Biener G, Niv A, Kleiner V and Hasman E 2002 Opt. Lett. 271875

[25] Born M and Wolf E 2005 Principles of Optics (Oxford: Pergamon)

[26] Trushkevych O, Ackerman P, Crossland W A and Smalyukh I I 2010 Appl. Phys. Lett. 97201906

[27] Ackerman P J, Qi Z, Lin Y, Twombly C W, Laviada M J, Lansac Y and Smalyukh I I 2012 Sci. Rep. 2414

[28] Hamdi R, Petriashvili G, Lombardo G, Santo M P D and Barberi R 2011 J. Appl. Phys. 110074902

[29] Marrucci L, Karimi E, Slussarenko S, Piccirillo B, Santamato E, Nagali E and Sciarrino F $2011 \mathrm{~J}$. Opt. 13064001 